\title{
REQUIREMENTS FOR COMPUTER-AIDED LEARNING FROM THE POINT OF VIEW OF ELECTRONIC DESIGN
}

\author{
Ricardo Reis \\ Instituto de Informática - Universidade Federal do Rio Grande do Sul, C.P 15064- CEP 91501- \\ 970 Porto Alegre, Brazil.E-mail: reis@inf.ufrgs.br
}

\begin{abstract}
The main challenge to set up an computer environment for microelectronics education (design or CAD) is to have a resourceful and specific framework and to made a consortium to fill this framework with tools that really use the advantages and facilities that a computer provides. Comparing to printed books, any figure should be transformed in a tool that could simulate the problem related. It also should allow the student interaction with. The learning flow of a computer-based course should also be different from traditional learning flows. The paper shortly extends this point proposing a new learning flow.
\end{abstract}

Key words: Electronic design education; CAD; computer aided learning; e-learning systems; standardization; semiconductor industry

\section{INTRODUCTION}

The existent information and communication technology provide the opportunity to supply tools to improve the learning process. But the availability of a new generation of computer-based education systems, that really use the available technology, is still fare away. Also, when we talk about computer-based education we should consider two situations: the first one, e-learning as a remote or self-learning system and a second situation where the computer-based system is used in a regular learning process inside an education institution. In both situations we have some similar specifications and needs, but we have also different ones. For example, in a 
remote education procedure where it is needed student evaluation or grading, it is needed a system that can do the certification that the right student is doing the exams. If we are using a computer-based system in a regular course in an educational institution we don't need a similar system, because the exams can be done in a specific room and time.

\section{ELECTRONIC DESIGN LEARNING}

The two main challenges in the development of an educational learning system are the learning framework itself and the development of the course contents. The learning framework can be divided in two parts: the administrative framework (that handles, for example, student access, student progress supervision) and the learning framework. The learning framework should control the learning flow by giving access to the modules related to a specific step of learning or to a specific learning module. The construction of the learning framework needs the definition of a standard for this framework. This standard should also guide the educators responsible for the development of the learning material. This a major task when we really construct a learning system that really use the computational facilities that are available nowadays. The development of the contents of a course must be divided between a large number of educators because the authoring of each learning module demands an enormous amount of work. We can easily conclude this if we compare a good learning system with a didactic book. We can accept that each static figure of a book could be replaced by an animated one when it is part of a computer-based learning system. For example, a figure of an atom in a book can be replaced by a simulation tool that emulates how an atom works. This "animated figure" can improve the learning process because it gives to the student a more real information of how an atom is composed and how it works. Another example, a figure of a transistor cut from a book should be replaced by an animated figure constructed as a simulation tool that shows how the $\mathrm{p}$ and $\mathrm{n}$ carriers moves in function of changes in the transistor input signal (gate signal). This tool could give an interactive experience for the student that could change the values of the gate signal and observe the possible changes in the flow of carriers.

So, for example, if we only consider the development of a basic computer-based microelectronic course based on a traditional book and if we change the figures by animated and interactive ones, the amount of authoring work is enormous and should be divided between several educators. As consequence, we need a standard to organize the process of construction and authoring a course that is made by different educators. It is also needs tools 
to help course construction by the educators (Indrusiak et al, 2003). A point that is not easy to solve is the different vocabulary between educators, but a tool could guide educators to construct texts with a more common vocabulary.

We also face another problem, how to set up a team of educators to construct a course, educators that probably work in different institutions around the world. The course framework must also consider that the educators and students could use different computer and operating system platforms. So, the standards must consider these different environments.

An Educational Modelling Language (EML) is also important to help educators to customize a course to a specific curriculum or to a specific student.

In the Electronic Design area we could divide the learning contents in several major modules. As example, one possible and simple division could be:

- microelectronic basics

- digital design

- analog design

- test

- CAD

Each one of these modules has some specific needs or characteristics. For example, the design of an integrated circuit demands the use of a big set of CAD tools. So, the students must have a good experience with the use of these CAD tools. The challenge is that the professional CAD systems are composed by a big set of tools that were developed during years by different teams of professionals, several times in different companies that were acquired along the years. There is also a lack of good tutorials to guide the student and designers in the learning process of how to use the CAD tools.

\section{COMPUTER-BASED LEARNING METHODS}

The use of computer-based learning systems in a regular course can change the approach or flow of learning. One of the problems that we can face in a course is the presence of students with different backgrounds. If we use a learning flow where as first step the student must do a self-learning procedure, with the use of a computer-based learning system, then the student with different backgrounds will take less or more time to learn the lesson but all the student could reach a same level of knowledge at the end of the lesson. This could be certified by a self-test done by the student himself. The professor could also remotely monitor the learning flow of each student. This an important feedback to the professor because he can know 
which points he should improve in the learning material, as well which parts he need to strengthen in the second step of the course. As a second step, the students could attend a course with the participation of a professor. In this second step the professor can consider that the students have the same knowledge about the subject. The students can put questions about the lessons they have studied by themselves and the educator can also go deep in the subject.

\section{LEARNING MODULE ANIMATION EXAMPLE}

One example of the substitution of a book figure by an animated object is the substitution of a figure representing a schematic at logic level by an animated object. It is possible to identify by colours the values of the inputs as well the voltage values in the connections (Fig. 1) (Casacurta et al, 2003). So, the student can change the value of an input by a click over it and he can observe the propagation of this input over the logic circuit by a change in the color of the connections belonging to a node which value changes in function of the input change.

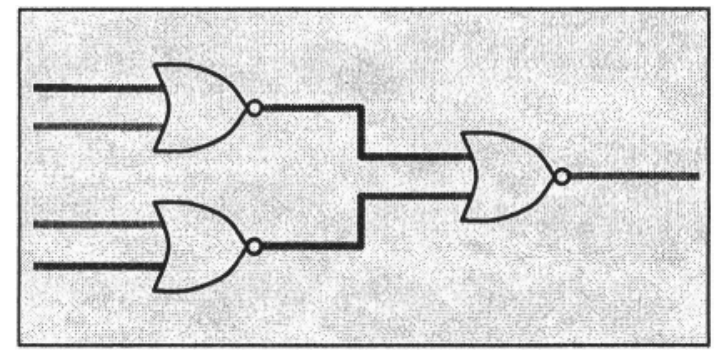

Figure 1. Example of logic circuit with inputs, output and connections colors related to voltage value (Casacurta et al, 2003)

Some features can be associated to this animated figure. It is possible to change the view of a logic cell or a group of logic cells to see its layout, where the colors of lines and regions are set in function of their voltage value (Fig. 2). This layout view simulates the view provide by an ElectronBeam microscope, but in color. It is also possible to control the velocity of propagation of input changes to help the understanding of signals propagation. This small example shows that a computer-based course can provide to the student a much better understanding when he has the help of animated objects that he can interact with. 


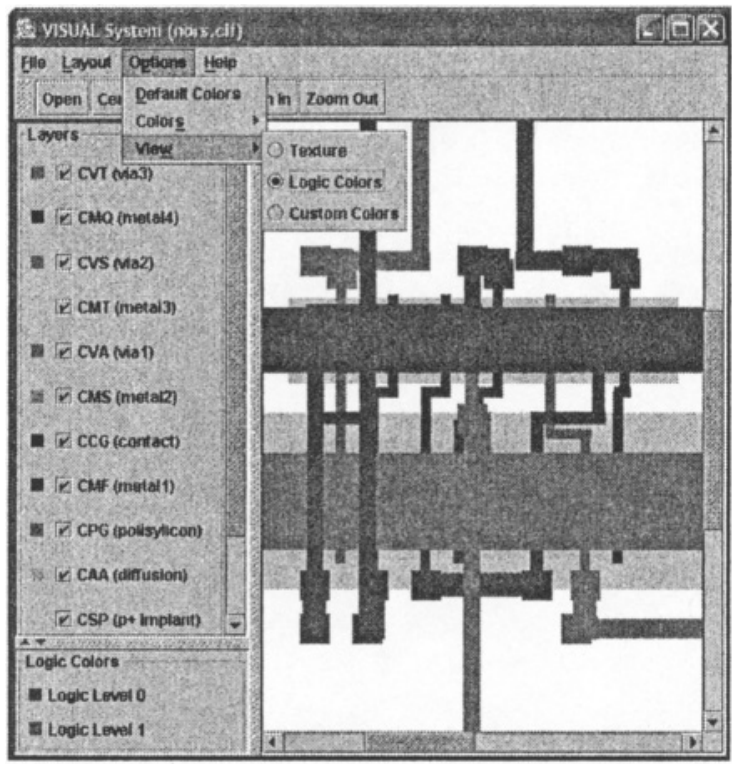

Figure 2. Example of circuit layout where connections and regions have colors related to their voltage value (Casacurta et al, 2003)

\section{CONCLUSIONS}

The development of computer-based learning systems demands standards as well frameworks to plug learning materials and to manage the learning flow. It is also necessary to define standards and tools to guide educators in the development and authoring of learning materials. These learning materials should really use the facilities that a computer can provide, like simulations and interactions with animated objects. A big challenge is to put together a large group of educators to prepare a course using a large set of animated figures and simulated objects.

A learning framework should work with any computer or operating system platform.

\section{ACNOWLEDGEMENTS}

We want to acknowledge Casacurta and Almeida for the work that they have done and that is used as example in section 4. 


\section{REFERENCES}

Indrusiak, L. S., Reis, R. A. L., Glesner, M., Alcántara G., Hörmann S., Steinmetz, R. Reducing Authoring Costs of Online Training in Microelectronics Design by Reusing Design Documentation Content. In: IEEE Microelectronics System Education, Anaheim, June 1-2, 2003. pp 57-58.

Casacurta, A., Almeida, M., Reis, R. , 2003, A Visual Simulation Tool at Layout Level, in: IEEE Microelectronics System Education, Anaheim, June 1-2, 2003, pp. 110-111. 